\title{
Mechanisms of pyrazinamide resistance in mycobacteria: importance of lack of uptake in addition to lack of pyrazinamidase activity
}

\author{
Catherine Raynaud, ${ }^{1}$ Marie-Antoinette Lanéelle, ${ }^{1}$ Ryan H. Senaratne, ${ }^{2}$ \\ Philip Draper, ${ }^{2}$ Gilbert Lanéelle ${ }^{1}$ and Mamadou Daffé ${ }^{1}$
}

\footnotetext{
1 Institut de Pharmacologie et de Biologie Structurale du Centre National de la Recherche Scientifique and Université Paul Sabatier, 205 route de Narbonne, 31077 Toulouse cedex, France

2 National Institute for Medical Research, Mill Hill, London NW7 1AA, UK
}

Author for correspondence: Mamadou Daffé. Tel: +33 5611755 69. Fax: + 33561175994. e-mail: daffe@ipbs.fr

Mycobacteria are known to acquire resistance to the antituberculous drug pyrazinamide (PZA) through mutations in the gene encoding pyrazinamidase (PZase), an enzyme that converts PZA into pyrazinoic acid, the presumed active form of PZA against bacteria. Additional mechanisms of resistance to the drug are known to exist but have not been fully investigated. Among these is the non-uptake of the pro-drug, a possibility investigated in the present study. The uptake mechanism of PZA, a requisite step for the activation of the pro-drug, was studied in Mycobacterium tuberculosis. The incorporation of $\left[{ }^{14} \mathrm{C}\right] \mathrm{PZA}$ by the bacilli was followed in both neutral and acidic environments since PZA activity is known to be optimal at acidic pH. By using a protonophore (carbonyl cyanide $\boldsymbol{m}$-chlorophenylhydrazone; (CCP), valinomycin, arsenate and low temperature, it was shown that an ATP-dependent transport system is involved in the uptake of PZA. Whilst the structurally analogous compound nicotinamide inhibited the transport system of PZA, other structurally related compounds such as pyrazinoic acid, isoniazid and cytosine did not. Acidic conditions were also without effect. Based on diffusion experiments in liposomes, it was found that PZA diffuses rapidly through membrane bilayers, faster than glycerol, whilst the presence of OmpATb, the porin-like protein of $M$. tuberculosis, in proteoliposomes slightly increased the diffusion of the drug. This finding may explain why the cell wall mycolate hydrophobic layer does not represent the limiting step in the diffusion of PZA, as judged from comparative experiments using a $\boldsymbol{M}$. tuberculosis strain and its isogenic mutant elaborating $\mathbf{4 0} \%$ less covalently linked mycolates. PZase activity, and PZA uptake and susceptibility in different mycobacterial species were compared. M. tuberculosis, a naturally PZA-susceptible species, was the only species that exhibited both PZase activity and PZA uptake; no such correlation was observed with the four naturally resistant species examined.

Mycobacterium smegmatis possessed a functional PZase but did not take up PZA; the reverse was true for the PZase-negative strain of Mycobacterium avium used, with PZA uptake comparable to that of $M$. tuberculosis.

Mycobacterium bovis BCG and Mycobacterium kansasii exhibited neither a PZase activity nor PZA uptake. These data clearly demonstrate that one of the mechanisms of resistance to PZA resides in the failure of strains to take up the drug, indicating that susceptibility to PZA in mycobacteria requires both the presence of a functional PZase and a PZA transport system. No correlation was observed between the occurrence and cellular location of PZase and of nicotinamidase in the strains examined, suggesting that one or both amides can be hydrolysed by other mycobacterial amidases.

Keywords: tuberculosis, Mycobacterium, pyrazinamide, pyrazinamidase, nicotinamide 


\section{INTRODUCTION}

Tuberculosis remains a major public health problem, claiming three million deaths annually in developing countries; the resurgence of the disease in industrialized countries and the emergence of multidrug-resistant mycobacteria have renewed interest in understanding the molecular mechanisms of drug resistance in these pathogens. In combination with the widely used frontline antituberculous drugs isoniazid (INH) and rifampicin, other drugs have been proved useful in the treatment of tuberculosis. Among these, pyrazinamide (PZA) has a special place in that it appears to kill a population of semi-dormant tubercule bacilli that are not affected by other antituberculous drugs (McCune $e t$ al., 1956), shortening the duration of treatment from the earlier norm of 12-18 months to the current standard of 6 months. Although PZA has long been known to be active in humans with pulmonary tuberculosis (Yeager, 1952) and is used in routine chemotherapy for tuberculosis, its mechanism of action is still obscure.

It was an early observation (Konno et al., 1967; McClatchy et al., 1981) that the resistance of clinical isolates of Mycobacterium tuberculosis to PZA correlates with the absence of an amidase activity, pyrazinamidase (PZase). This enzyme transforms PZA into pyrazinoic acid (POA), a substance which is supposed to be the active molecule against $M$. tuberculosis, even though no target has yet been experimentally identified. Indeed, tubercle bacilli are not inhibited by PZA when tested in vitro in a neutral environment, whilst they become susceptible to the drug when tested in an acidic environment in vitro (McDermott \& Tompsett, 1954) or within monocytes (Mackaness, 1956). Furthermore, the gene encoding $M$. tuberculosis PZase ( $p n c A$ ) has been cloned recently and mutations in $p n c A$ were identified in both acquired PZA-resistant clinical isolates of $M$. tuberculosis and naturally PZA-resistant strains of Mycobacterium bovis (Scorpio \& Zhang, 1996). Transformation of these strains with a functional $p n c A$ gene restored PZase activity and PZA susceptibility (Scorpio \& Zhang, 1996). Whilst the above data clearly establish that PZase is involved in the action of the pro-drug PZA, other published observations remain to be explained. For instance, transformation with the $p n c A$ gene of a PZase-positive strain of Mycobacterium avium conferred PZA susceptibility to PZA-resistant M. tuberculosis complex organisms, indicating that resistance to the drug is not due only to the mutations in $p n c A$ (Sun et al., 1997). Likewise, some strains of M. tuberculosis (Butler \& Kilburn, 1983), Mycobacterium smegmatis and M. avium (Konno et al., 1967) possess a PZase activity but are resistant to PZA, further demonstrating the existence of alternative mechanisms of resistance to PZA.

In addition to mutations in genes encoding the activation of drugs, micro-organisms are known to have developed several other strategies to resist being killed by chemotherapeutic agents (Davies, 1994; Neu, 1992; Nikaido, 1994; Spratt, 1994). These include mutations in the genes encoding drug targets, lack of or a decrease in transport of drugs, the operation of efflux pumps and the production of drug-inactivating enzymes. In the case of PZA, no such mechanism is known to occur and PZA uptake by mycobacteria has not been studied to date. Consequently, as an attractive hypothesis, we examined the possibility that mycobacterial resistance to PZA may be due to the lack of uptake of the pro-drug. We first studied the uptake of PZA in the PZA-sensitive strain H37Rv of M. tuberculosis, and then compared it to that occurring in four mycobacterial species differing from one another by their PZase activity and susceptibility to the drug.

\section{METHODS}

Mycobacterial strains and cultures. $M$. bovis BCG (Institut Pasteur, Paris; CIPT 140040001), Mycobacterium kansasii (ATCC 12478) and M. tuberculosis H37Rv (ATCC 27294), and M. tuberculosis Mt103 and its isogenic mutant MYC1554, obtained from an ordered M. tuberculosis transposon mutagenesis library and shown to have an inactivated $85 \mathrm{C}$ gene (number Rv8303, gene $f b p C 1$ ) due to the insertion of a transposon (Jackson et al., 1999), were inoculated by loop (roughly $10 \mathrm{mg}$ wet wt bacteria) into $100 \mathrm{ml}$ Sauton's medium (Sauton, 1912) in $250 \mathrm{ml}$ flasks and incubated at $37^{\circ} \mathrm{C}$ as surface pellicles for 2-4 weeks. A PZA-negative strain of $M$. avium (ATCC 15769) and M. smegmatis $\mathrm{mc}^{2} 155$ were grown with shaking on 7H9 Middlebrook broth (Difco) enriched with $0.5 \%$ Casitone and containing $0.2 \%(\mathrm{w} / \mathrm{v})$ glyc ol at $37^{\circ} \mathrm{C}$.

Tritium labelling of bacteria. Background labelling of bacterial cells by $\left[{ }^{3} \mathrm{H}\right]$ uracil was used to solve the problem of quantification of the mycobacterial biomass, one of the wellknown experimental difficulties with these organisms since they usually form large clumps in culture. Thus, the problem was solved by relating the counts of the ${ }^{14} \mathrm{~S}$-labelled compound to those of the $\left[{ }^{3} \mathrm{H}\right]$ uracil labelling (Bardou et al., 1998). Bacteria were harvested by pouring off the medium and dispersed by gentle shaking with glass beads (Ortalo-Magné et al., 1995). Declumped cells were resuspended in $20 \mathrm{ml}$ fresh Sauton's medium containing $\left[5,6-{ }^{3} \mathrm{H}\right]$ uracil $\left(0 \cdot 37 \mathrm{MBq} \mathrm{ml}^{-1}\right)$; the bacterial suspensions were sedimented for $10 \mathrm{~min}$ to remove clumps and the supernatants were collected for overnight growth. At least $90 \%$ of the mycobacteria are individualized by this method and the remaining $10 \%$ form small aggregates containing two or three bacilli (N'Diaye et al., 1998). Labelled cells were collected by centrifugation $(3000 \mathrm{~g}, 10 \mathrm{~min})$, washed and suspended in the transport buffer (HEPES, $\mathrm{pH} 7 \cdot 2$ ). In some experiments, aliquots of the cell suspension were dried, weighed and counted to correlate ${ }^{3} \mathrm{H}$-labelling with cell dry weight.

Transport. Transport measurement was carried out in a $1 \mathrm{ml}$ assay in HEPES buffer ( $25 \mathrm{mM}, \mathrm{pH} 7 \cdot 2)$ without added carbon source, except when otherwise stated, at room temperature $\left(23^{\circ} \mathrm{C}\right)$, under continuous shaking. These correspond to optimal conditions for transport measurements, as previously determined (Bardou et al., 1998). Each assay contained between 30 and $40 \mathrm{mg}$ cell dry weight and $3.85 \times 10^{-5} \mathrm{M}$ $\left[{ }^{14} \mathrm{C}\right] \mathrm{PZA}$. To minimize hazard, one of the experimental difficulties with studying transport using pathogenic mycobacterial species such as $M$. tuberculosis, cells were centrifuged through silicone oil instead of using the conventional filtration method to separate the labelled cells from the transport medium (Bardou et al., 1998). Aliquots of $0.1 \mathrm{ml}$ 
were taken at timed intervals, layered on the surface of Eppendorf tubes containing $0.25 \mathrm{ml}$ oil (silicone oil/paraffin oil, $1:(0 \cdot 2, \mathrm{v} / \mathrm{v})$ and immediately centrifuged at $13000 \mathrm{~g}$ for $1 \mathrm{~min}$ as previously described (Bardou et al., 1998). Centrifuge tubes were rapidly frozen in dry ice and the pellets recovered by cutting off the conical tips of the tubes into the counting vial. Forty min sonication in a bath was necessary to disperse the pellet in the scintillation liquid. Counting was performed on a Packard Tricarb 1900 TR scintillation counter using a ${ }^{3} \mathrm{H} /{ }^{14} \mathrm{C}$ program. Uptake determinations were repeated in at least three independent experiments. To distinguish the inherent and rapid labelling associated with binding to the outer cell envelope from transport, i.e. the labelling inside the cells, uptake values were corrected for the residual radioactivity at time zero (the time at which centrifugation was started).

When used, arsenate $(50 \mathrm{mM}$ final concentration) and carbonyl cyanide $m$-chlorophenylhydrazone (CCCP; $10^{-5} \mathrm{M}$ final concentration) were added $15 \mathrm{~min}$ before starting the uptake experiments. Experiments at $4{ }^{\circ} \mathrm{C}$ were performed with cells preincubated for $1 \mathrm{~h}$ in melting ice.

Determination of PZase activity. This was done according to Bönicke (1962) as modified by Wayne (1974). About $15 \mathrm{mg}$ (wet wt) cells, $5 \mathrm{mg}$ crude bacterial extracts (prepared as previously described by Raynaud et al., 1998), or $0.3 \mathrm{ml}$ culture filtrate was incubated at $37^{\circ} \mathrm{C}$ with $20 \mu \mathrm{PZA}(64 \mathrm{mM})$ for $24 \mathrm{~h}$, prior to the addition of $0 \cdot 2 \mathrm{ml}$ freshly prepared ferrous ammonium sulfate $(1 \%, \mathrm{w} / \mathrm{v}$, in water). The mixture was refrigerated for $4 \mathrm{~h}$ before use. The appearance of a pink colour indicated the release of POA. Fresh Sauton's medium was used as negative control.

Production of culture filtrates. The pellicle growth conditions enabled cells to be easily harvested by permitting the medium to be collected whilst the pellicles remained attached to the flasks. The culture medium was sterilized by filtration through a $0.2 \mu \mathrm{m}$ Nalgene filter (Nalge) and concentrated under vacuum to one-tenth of the initial volume. Protein concentration was determined by the Coomassie blue reaction (Bio-Rad protein assay) and filtrates were adjusted to $100 \mu \mathrm{g}$ protein $\mathrm{ml}^{-1}$ by dilution.

Isolation of surface-exposed materials. Surface-exposed materials were recovered from the harvested bacteria as previously described (Ortalo-Magné et al., 1995). Mycobacterial cells were gently shaken for 1 min with $10 \mathrm{~g}$ glass beads $(4 \mathrm{~mm}$ diameter) per $2 \mathrm{~g}$ (wet wt) cells. The resulting declumped cells were suspended in distilled water $(50 \mathrm{ml}$ per flask) and the material removed from the bacteria was immediately sterilized by filtration as described above. The preparations were concentrated under vacuum to adjust their protein concentration to $50 \mu \mathrm{g} \mathrm{ml}^{-1}$.

Diffusion into liposomes and proteoliposomes. Liposomes were prepared with $57 \mu \mathrm{mol}$ phosphatidylcholine and $2 \cdot 3 \mu \mathrm{mol}$ phosphatidylglycerol. Optical density changes in liposome suspensions were measured with a Uvikon UV 333 spectrophotometer; recorded data were converted to commaseparated value files as previously described (Senaratne $e t$ al., 1998). The liposome swelling assays in isotonic solutions $(25 \mathrm{mM})$ of sucrose, glycerol and PZA were followed over a 2 min period. A decrease in the optical density corresponds to swelling of liposomes. Porin-containing liposomes (proteoliposomes) were prepared and assayed as previously described (Senaratne et al., 1998); up to $25 \mu \mathrm{g}$ porin (OmpATb) was used to prepare proteoliposomes.

Chemicals. $\left[{ }^{14} \mathrm{C}\right] \mathrm{PZA}\left(52 \mathrm{mCi} \mathrm{mmol}^{-1} ; 1.92 \mathrm{GBq}^{\mathrm{mmol}}{ }^{-1}\right)$ was a generous gift of the National Institute of Health (USA), NIH
AIDS Research and Reference Program (NIH, Manassas, VA, USA). $\left[{ }^{3} \mathrm{H}\right]$ Uracil $\left(1.85 \mathrm{TBq} \mathrm{mmol}^{-1}\right)$ was from Amersham. Silicone oil DC550 was from Serva $\left(d\right.$ at $25^{\circ} \mathrm{C}=1 \cdot 07$; Boehringer Mannheim) and codex paraffin oil from Gifrer. The scintillation solution Aqualuma was obtained from Lumac. All other chemicals were from Sigma.

\section{RESULTS}

\section{Localization and distribution of PZase in M. tuberculosis and other mycobacteria}

PZA is assumed to be a pro-drug that needs to be converted into POA by PZase in order to show bactericidal activity against $M$. tuberculosis and other susceptible mycobacteria. Thus, the choice of the compound to be used for uptake experiments depends on the location of PZase. If the enzyme is surfacelocated, there is no need to follow the uptake of PZA by bacilli since the drug would be activated to POA before its uptake. Consequently, whole cells as well as the materials extracted by the gentle mechanical treatment of M. tuberculosis cells and from four other mycobacteria (Ortalo-Magné et al., 1995) were analysed for their PZase activity throughout the bacterial growth phases. In addition, the corresponding culture filtrates were analysed for both isocitrate dehydrogenase, a cytoplasmic enzyme that is only released by lysed cells, and PZase since false positive results may be obtained due to bacterial lysis (Raynaud et al., 1998). The variability in the reported literature data on the detection of mycobacterial degradation products of amides, including PZA, was attributed by Tarnok \& Röhrscheidt (1976) to the use of non-optimal substrate concentration. Using the recommended optimal concentration $(4 \mathrm{mM})$ of PZA, PZase activity was not detectable in any of the surface-exposed materials examined whilst easily found in whole cells of M. tuberculosis (positive control), as expected. PZase activity was neither detected in whole cells nor in the bacterial cell lysates from three out of the four PZA-resistant strains tested, namely M. bovis BCG, M. avium $(50-80 \%$ of strains belonging to this species are recorded in the literature as being PZA-positive) and $M$. kansasii, but was found in both whole cells and lysate of the fourth PZA-resistant strain, M. smegmatis (Table 1). Thus, the lack of detection of enzyme activity in some of the strains is probably due to loss of enzyme activity rather than an insensitive nature of the enzyme assay used since PZase-positive mycobacterial strains, including $M$. avium, are easily detectable using whole cells (see, for example, Tacquet et al., 1967; Tsukamura \& Tsukamura, 1966). It follows then that PZase is not located on the surfaces of the mycobacteria examined but may be present in inner compartments of both PZAsusceptible and -resistant bacterial cells.

\section{PZA uptake by M. tuberculosis H37Rv}

Since the mycobacterial PZase is not surface-located, PZA has to be taken up by the bacterial cells and converted into POA, its presumed active form. As PZA is known to be inactive against the tubercle bacillus 
Table 1. PZA uptake by and PZase and nicotinamidase activities of some mycobacteria

PZase and nicotinamidase activities were determined in the culture filtrates (devoid of lysis markers), bead-extracted materials and cell sonicates from the species examined as described in the Methods section and by Raynaud et al. (1998), respectively. When the activity was detected in the cell sonicates but not in the extracellular and surface-exposed materials, the enzyme was considered as having an internal location. PZA uptake was determined using $\left[{ }^{14} \mathrm{C}\right] \mathrm{PZA}$ (see Fig. 5).

\begin{tabular}{|lccc|}
\hline Strains & PZase* & Nicotinamidase* & PZA uptake $\dagger$ \\
\hline M. tuberculosis H37Rv & I & E & + \\
M. smegmatis $\mathrm{mc}^{2}$ 155 & I & I & - \\
M. bovis BCG Pasteur & - & E & - \\
M. kansasii (ATCC 12478) & - & I & $+\neq$ \\
M. avium (ATCC 15769) & - & ND & + \\
\hline
\end{tabular}

*E, Extracellular location; I, internal location; ND, not determined; -, no enzyme activity detected. $t+$, PZA uptake observed; -, no PZA uptake observed (see Fig. 5).

$\ddagger$ This strain takes up PZA but at a lower rate than M. tuberculosis and M. avium (see Fig. 5).

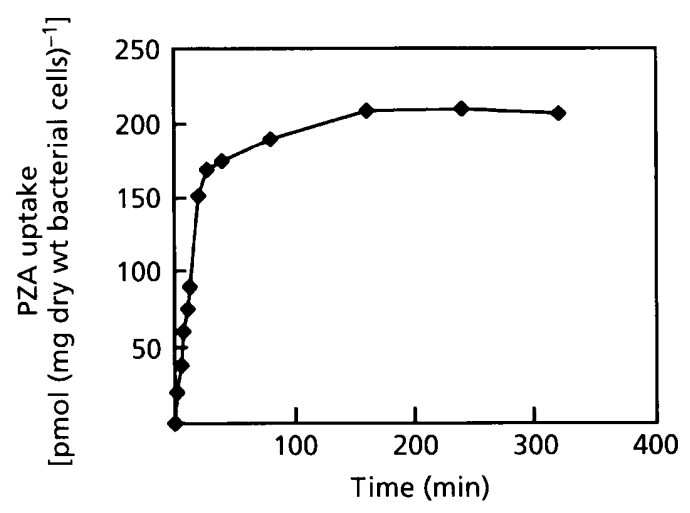

Fig. 1. PZA uptake kinetics in $M$. tuberculosis H37Rv.

under the normal neutral $\mathrm{pH}$ culture conditions and active in acidic (pH 5.5) medium (McDermott \& Tompsett, 1954), the uptake of the drug was followed at both $\mathrm{pH} 5.5$ and $7 \cdot 5$. Interestingly, no obvious difference was observed between the accumulation of radiolabelled PZA by M. tuberculosis in the two $\mathrm{pH}$ conditions, both in terms of initial uptake velocity and accumulation plateau, suggesting that the observed higher efficiency of the drug at acidic $\mathrm{pH}$ is not due to an increase in uptake of the drug. The drug was rapidly taken up by the cells for the first $20 \mathrm{~min}$, followed by a plateau phase; assuming a bacterial cell volume of 2.4-3.0 $\mu \mathrm{l}$ (mg dry wt) $)^{-1}$ (Wang \& Takayama, 1972; Youatt \& Tham, 1969) at the plateau the drug was concentrated 5.4-6.2fold within cells (Fig. 1).

We recently showed that $\mathrm{INH}$, a structural analogue of nicotinamide and PZA, enters $M$. tuberculosis cells by passive diffusion (Bardou et al., 1998). Unlike the two other transport systems used by cells to achieve the uptake of compounds, i.e. facilitated diffusion or active transport, no protein is implicated in passive diffusion of molecules. Thus, we first looked for this latter phenomenon in the transport of PZA by performing the transport experiments in melting ice, an experimental condition known to abolish both facilitated diffusion and active transport. In contrast to what was observed for INH, the uptake of PZA was completely abolished at $4{ }^{\circ} \mathrm{C}$ (Fig. 2a), as previously shown for the uptake of glycerol, a molecule that enters cells by facilitated diffusion (Lin, 1976). Consistent with the involvement of a transport system, the addition of a tenfold concentration of non-labelled PZA also inhibited the accumulation of radioactive PZA (Fig. 2b), demonstrating that the uptake system can be saturated, as expected for a transport system. The possibility that PZA is taken up by a transport system devoted to nicotinamide, a structural analogue of PZA, was investigated by adding a tenfold concentration of non-labelled nicotinamide. As shown in Fig. 2b, the addition of nicotinamide also resulted in the inhibition of the uptake of $\left[{ }^{14} \mathrm{C}\right] \mathrm{PZA}$. The structural analogues POA (in both acidic and neutral conditions), INH and cytosine, as well as the unrelated glucose, did not compete for the uptake of the drug (data not shown), pointing to the specificity of the transport system. It was thus concluded that the uptake of PZA occurs via a selective transport system.

To further characterize the transport sytem involved in the uptake of PZA, we investigated the possible involvement of energy dependency in the system by testing the effects of two molecules known to inhibit ion-driven transport in mycobacteria (Prasad et al., 1976; Bardou et al., 1998). Thus, a protonophore (CCCP) acting on the transmembrane proton electrochemical potential $\left(\Delta \bar{\mu}_{\mathrm{H}^{+}}\right)$ and known to completely inhibit the uptake of proline at $10^{-5} \mathrm{M}$ (Bardou et al., 1998), or valinomycin in the presence of $50 \mathrm{mM} \mathrm{KCl}$, which reduces transmembrane potential, were added to the bacterial suspensions $15 \mathrm{~min}$ before the labelling experiments. Neither of these conditions modified the uptake of PZA, demonstrating that the PZA and nicotinamide uptake system is not coupled to an ion gradient (data not shown). In sharp contrast, the addition of $50 \mathrm{mM}$ arsenate, known 


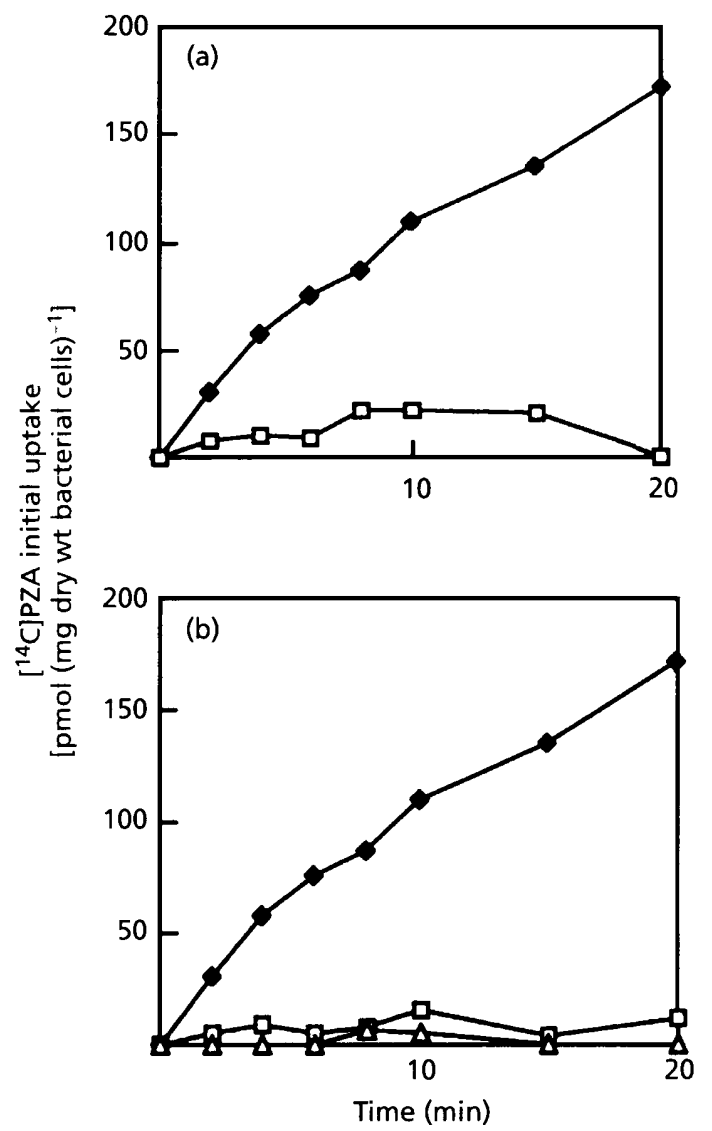

Fig. 2. Effect of low temperature and of competitors on $\left[{ }^{14} \mathrm{C}\right] \mathrm{PZA}$ initial uptake. (a) $\diamond$, Control uptake at room temperature; $\square$, uptake at melting ice temperature. (b) $\diamond$, Control uptake $\left(3.85 \times 10^{-5} \mathrm{M}\left[{ }^{14} \mathrm{C}\right] \mathrm{PZA}\right) ; \square$, uptake of $3.85 \times 10^{-5} \mathrm{M}\left[{ }^{14} \mathrm{C}\right] \mathrm{PZA}$ in the presence of $3.85 \times 10^{-4} \mathrm{M}$ nonradioactive $P Z A ; \triangle$, uptake of $3.85 \times 10^{-5} \mathrm{M}\left[{ }^{14} \mathrm{C}\right] \mathrm{PZA}$ in the presence of $3.85 \times 10^{-4} \mathrm{M}$ nicotinamide.

to deplete ATP in mycobacterial cells (Prasad et al., 1976), resulted in the reduction of the initial uptake velocity of PZA by a factor of two (Fig. 3). Since this concentration of arsenate has no effect on PZase activity (unpublished results), it was obvious that an ATPdependent transport system located in the plasma membrane is involved in the uptake of PZA; however, based on the observed data, it is not possible to discriminate between an active primary transport system using ATP as energy source and a facilitated transport followed by a metabolic step involving ATP.

\section{Diffusion of PZA through the outer envelope of M. tuberculosis}

Mycobacteria possess a complex cell envelope composed of a capsule, a cell wall and a plasma membrane (Daffé \& Draper, 1998). Entry of metabolites and drugs into the bacterial cell can be divided into two steps: (i) diffusion through the outer envelope (capsule, cell wall); and (ii) penetration through the plasma membrane into the cell cytoplasm. The uptake initial velocity is an overall parameter that is related to the velocity of the

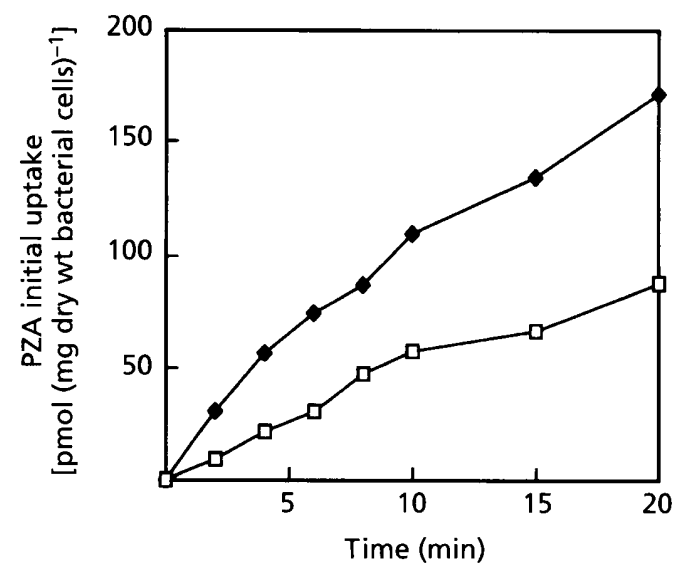

Fig. 3. Effects of arsenate, an inhibitor of the production of ATP, on PZA initial uptake. $\bullet$, Control uptake; $\square$, uptake in the presence of $50 \mathrm{mM}$ arsenate.

slowest step of the above process. It is currently assumed that the mycolic acids that are covalently linked to the cell wall arabinogalactan represent the basis of a hydrophobic outer layer in mycobacteria which presents some analogies with the outer membrane of Gramnegative bacteria (Daffé \& Draper, 1998; Brennan \& Nikaido, 1995). As this layer may represent a limiting step for diffusion of PZA, a water-soluble molecule, we first compared the uptake of glycerol, a well-known hydrophilic substance used by mycobacteria as a carbon source, by two isogenic strains of $M$. tuberculosis, a clinical isolate (Mt103) and its insertional mutant (MYC1554) defective in the production of antigen 85C, one of the mycoloyl transferases of $M$. tuberculosis (Belisle et al., 1997). The mutant elaborates $40 \%$ less covalently linked mycolic acids and was shown to take up glycerol with an initial velocity significantly higher than the parent strain (Jackson et al., 1999), validating the existence of the postulated hydrophobic barrier. This pair of strains was then used to determine the influence of the hydrophobic layer of the outer envelope on the uptake of PZA. In contrast to glycerol (Jackson et al., 1999), no difference was observed between the uptake of PZA by the two isogenic $M$. tuberculosis strains (data not shown), suggesting that the diffusion of glycerol through the outer envelope of M. tuberculosis, but not that of PZA, represents a limiting step in the uptake of the molecule.

The difference in the diffusion pattern of the two molecules in the outer cell envelope layer may be the consequence of the higher hydrophilicity of glycerol, resulting in a slower diffusion of this molecule through the hydrophobic barrier. To test this hypothesis, the diffusion of the two molecules was followed through a model hydrophobic membrane, multilayered vesicles of phosphatidylcholine. Such vesicles (liposomes) suspended in an isotonic solution of a diffusable molecule swell owing to a compensatory entry of water. Using $25-30 \mathrm{mM}$ of the different compounds, a con- 

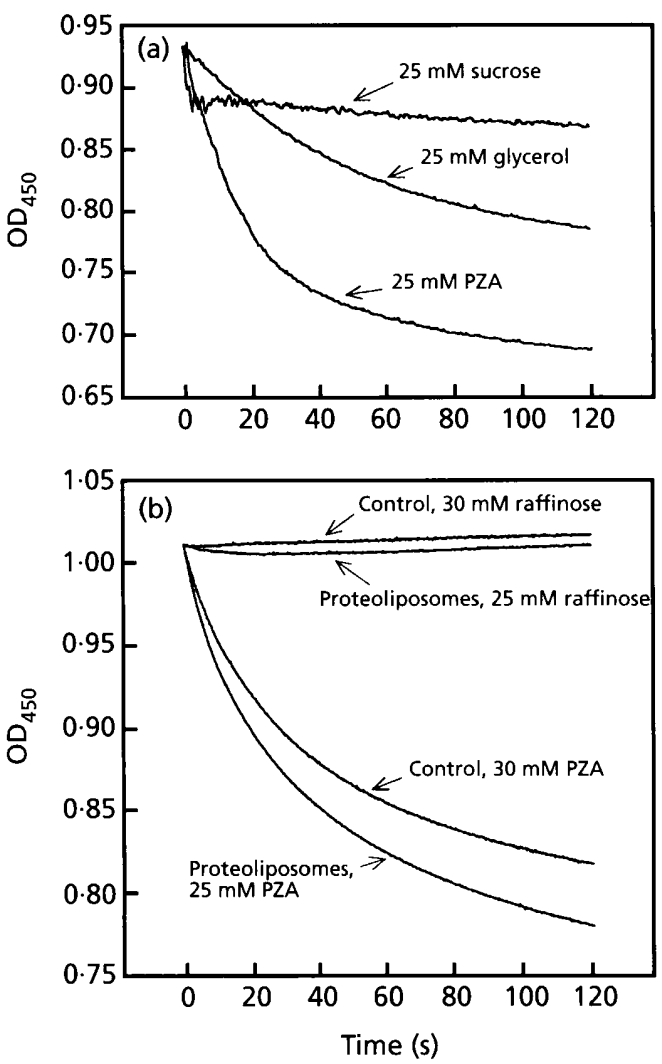

Fig. 4. PZA diffusion into liposomes and proteoliposomes. Sucrose or raffinose were used as negative controls (negligible diffusion) and glycerol as a positive control for diffusion (very hydrophilic small molecule). (a) Diffusion into multilayered liposomes; (b) diffusion into proteoliposomes containing recombinant OmpATb of $M$. tuberculosis. A decrease in $\mathrm{OD}_{450}$ corresponds to swelling of the vesicles. The slight irregularity in the plots is attributable to instrument noise.

centration much higher than the $10^{-5} \mathrm{M}$ used in the uptake assays with whole bacteria but necessary for diffusion experiments into liposomes, the initial swelling velocity of liposomes was much faster in an isotonic solution of PZA than in that of glycerol (Fig. 4a), as postulated. Although these results with liposomes can not be extrapolated to those obtained in uptake assays with whole bacteria due to the range of concentrations used, they clearly demonstrated that PZA has a greater diffusibility than glycerol through a hydrophobic layer.

Because the hydrophilic PZA may also enter mycobacteria through the OmpATb-porin-like proteins recently shown to be present in the cell walls of $M$. tuberculosis (Senaratne et al., 1998), the contribution of OmpATb to the uptake of PZA was investigated. The presence of OmpATb slightly increased the diffusion of PZA into liposomes (Fig. 4b); the significance of the difference is, however, not clear. Nevertheless, it appeared that the occurrence of porin in the outer cell envelope does not greatly affect the intrinsic high diffusion rate of PZA through hydrophobic barriers and that diffusion of the molecule through the outer envelope

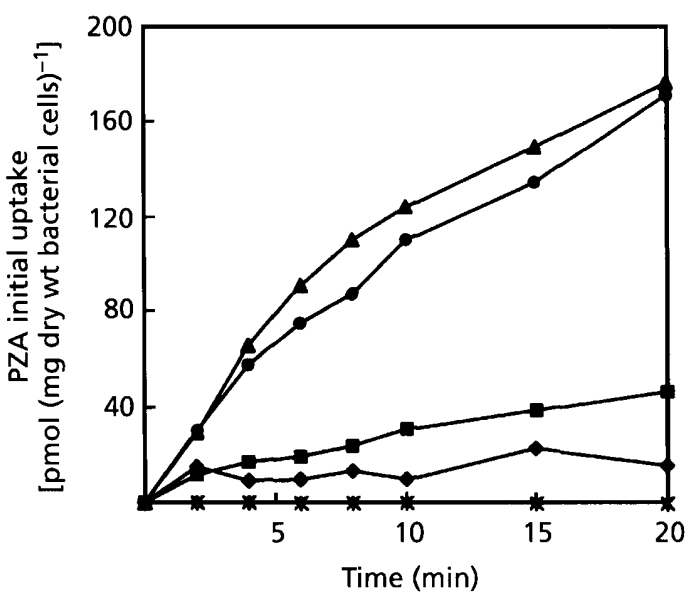

Fig. 5. PZA initial uptake in several mycobacterial species 0. $M$. tuberculosis H37Rv; $\boldsymbol{A}, M$. avium ATCC 15769; $M$. kansasii ATCC 12478; $\bullet, M$. bovis BCG strain Pasteur; $*$, M. smegmatis $\mathrm{mc}^{2} 155$.

of M. tuberculosis is not the limiting step in the uptake of the drug, consistent with the observed lack of increase of the uptake of PZA in the mutant having $40 \%$ less cellwall-linked mycolic acids in the outer layer (see above).

\section{PZA uptake by naturally PZA-resistant mycobacteria}

As functionally active PZase may be produced by some naturally resistant mycobacterial species (Table 1), the uptake of the drug, a prerequisite for its activation into POA, by these bacilli was investigated. As shown in Fig. 5 , only two out of the four strains examined showed a significant uptake of PZA; the PZA-negative strain of $M$. avium we studied exhibited the same initial uptake velocity as $M$. tuberculosis whereas M. kansasii showed a much lower velocity for the uptake of the drug. In contrast, neither M. bovis BCG nor M. smegmatis took up PZA. In the same experimental conditions, the latter species transported glycerol in a fashion similar to that of M. tuberculosis and PZase activity was demonstrated in the M. smegmatis sonicate (data not shown). It follows then that not all mycobacterial strains have a transport system able to take up PZA.

\section{DISCUSSION}

The present study was undertaken in order to explain some of the resistance mechanisms of mycobacteria to PZA and focussed on the uptake of the drug by bacterial cells, an important issue that has not been investigated previously. We first demonstrated that PZase, the enzyme that catalyses the conversion of PZA into the bactericidal POA, is not exposed on the bacterial surface, implying that the drug has to gain entry into the cells prior to its activation. This entry is ATP-dependent as proved by the inhibition of the uptake of PZA by the addition of arsenate and assays in low temperature conditions; it was not possible, however, to discriminate 
between an ATP-driven active transport through the plasma membrane from a facilitated diffusion through the cell envelope followed by an ATP-dependent step. As suggested by competition experiments, PZA uses the system naturally devoted to the transport of the physiologically important nicotinamide molecule. In Escherichia coli, uptake of nicotinamide is reduced by inhibitors of glycolysis and uncouplers of ATP production (Tritz, 1987), a result consistent with our observation in M. tuberculosis.

To reach its activation site, PZA has to diffuse through the complex mycobacterial cell envelope, which contains, in addition to the conventional plasma membrane, a hydrophobic barrier composed of cell wall mycolates (Daffé \& Draper, 1998; Brennan \& Nikaido, 1995). From the data obtained on liposomes, proteoliposomes and the M. tuberculosis mutant defective in the synthesis of one mycoloyl transferase, it appeared that the diffusion of PZA through the outer cell layers was not the limiting step of the overall process of its uptake.

M. tuberculosis, in contrast to many other mycobacteria, is susceptible to PZA and the acquired resistance of clinical isolates to the drug has been proved to be commonly due to mutations in the $p n c A$ gene, which encodes the activating enzyme PZase (Scorpio \& Zhang, 1996). However, no mutation in the pncA gene has been found in some strains of M. tuberculosis with acquired PZA resistance (Scorpio et al., 1997) and different naturally PZA-resistant mycobacterial species have been found to possess PZase (Konno et al., 1967). The best example of the latter category is the PZAresistant strain of $M$. avium which has been shown to possess a functionally active $p n c A$ gene (Sun et al., 1997). It followed then that further mechanisms of resistance remained to be elucidated. One of these was identified in the present study as being the failure of PZA uptake by resistant strains. This conclusion is supported by the expected observation that $M$. smegmatis, which does not take up PZA, is resistant to the drug. We also noted that M. tuberculosis was the only PZA-susceptible strain and exhibited both PZA uptake and PZase activity; all the naturally PZA-resistant strains examined here were either PZase-negative (M. avium and $M$. kansasii), failed to transport PZA (M. smegmatis) or were deficient in both functions (M. bovis BCG). We thus conclude that a PZA-susceptible strain should possess both a PZase activity and a membrane transport system accepting PZA as substrate. It is likely that PZA can passively diffuse through membranes devoid of a nicotinamide transport system but this phenomenon would be detectable only at high PZA concentrations. The corresponding strains would be PZA-sensitive at high PZA concentrations, provided that they exhibit a sufficient PZase activity.

Early studies on the mechanisms of action of PZA and the mechanisms of resistance of $M$. tuberculosis to the drug prompted the suspicion that there is a single enzyme with nicotinamidase activity but capable of reacting with the foreign substance, PZA (Konno et al., 1967). The structural similarity between PZA and nicotinamide, and the fact that nicotinamide also inhibits the growth of $M$. tuberculosis at low $\mathrm{pH}$, supported this assumption. In addition, resistance to either drug was accompanied by resistance to the other (Konno et al., 1967). Furthermore, transformation with the pncA gene of a PZase-positive but naturally PZA-resistant strain of $M$. avium, conferred PZA susceptibilty to PZA-resistant $M$. tuberculosis complex organisms (Sun et al., 1997). Thus, it is currently assumed that the mycobacterial enzyme possesses both nicotinamidase and PZase activities. This assumption is, however, in apparent conflict with the enzyme activities shown in Table 1 . Whilst all the strains studied exhibited a nicotinamidase activity located in various cell envelope compartments (Raynaud et al., 1998; this work) only $M$. tuberculosis and $M$. smegmatis showed a detectable PZase activity. In addition, whilst nicotinamidase activity was found to be extracellular and/or surfaceexposed in both $M$. tuberculosis and M. bovis BCG (Raynaud et al., 1998), PZase activity was neither detectable in the outermost capsular components of $M$. tuberculosis nor in whole cells and the bacterial lysate of $M$. bovis BCG. The failure to correlate nicotinamidase and PZase activities could be attributable to different sensitivities of the tests used. However, this hypothesis is unlikely since we used $4 \mathrm{mM}$ concentrations of nicotinamide and PZA, i.e. at least tenfold the highest $K_{m}$ determined for the two amides in mycobacteria (Boshoff \& Mizrahi, 1998); using this optimal condition, Tarnok \& Röhrscheidt (1976) also found both nicotinamidasenegative/PZase-positive and nicotinamidase-positive/ PZase-negative strains among the mycobacteria they examined, demonstrating that the failure to detect one of the enzyme activities was not due to the lack of sensitivity of the tests used. Thus, our data may be explained by the occurrence of more than one amidase able to hydrolyse nicotinamide in mycobacteria, one of which can also hydrolyse PZA. This hypothesis is also supported by several earlier observations, among them the fact that $M$. kansasii can hydrolyse nicotinamide but not PZA (Tacquet et al., 1967) whereas 'Mycobacterium thamnopheos' hydrolyses PZA but not nicotinamide (Bönicke, 1960) and 'Mycobacterium balnei' will grow on PZA as nitrogen source but not on nicotinamide (Tsukamura \& Tsukamura, 1966). A parallel of the proposed phenomenon would be the mycobacterial catalases: different species possess one or more catalases (Bartholomew, 1968; Quémard et al., 1995; Wayne \& Diaz, 1988) and one of these has an additional peroxidase activity. Further studies on these poorly studied mycobacterial amidases are warranted.

\section{ACKNOWLEDGEMENTS}

The authors are grateful to Dr Brigitte Gicquel (Institut Pasteur, Paris, France) for providing strains Mt103 and MYC1554 and to Miss Estelle Dransart (Université Paul Sabatier, Toulouse, France) for excellent technical assistance for the liposome swelling assays. This work is supported in 
part by a grant (no. 97002346) from the Région Midi-Pyrénées (Toulouse, France). The gift of radiaoactive PZA by the National Institute of Allergy and Infectious Diseases through the NIH AIDS Research and Reference Program is gratefully acknowledged.

\section{REFERENCES}

Bardou, F., Raynaud, C., Ramos, C., Lanéelle, M. A. \& Lanéelle, G. (1998). Mechanism of isoniazid uptake in Mycobacterium tuberculosis. Microbiology 144, 2539-2544.

Bartholomew, W. R. (1968). Multiple catalase enzymes in two species of mycobacteria. Am Rev Respir Dis 97, 710-712.

Belisle, J. T., Vissa, V. D., Sievert, T., Takayama, K., Brennan, P. J. \& Besra, G. S. (1997). Role of the major antigen of Mycobacterium tuberculosis in cell wall biogenesis. Science 276, 1420-1422.

Bönicke, R. (1960). Über das Vorkommen von Acylamidasen in Mycobakterien. IV. Die Differenzierung schnell wachsender Mycobakterien der Species Mycobacterium smegmatis, Mycobacterium phlei, Mycobacterium fortuitum und Mycobacterium thamnopheos durch Nachweis ihrer unterschiedlichen acylamidatischen Stoffwechselleistungen. Zentbl Bakteriol Parasitenkd Infektkrankh Hyg Abt I Orig 179, 209-223.

Bönicke, R. (1962). L'identification des mycobactéries à l'aide de méthodes biochimiques. Bull Int Union Tuberc 32, 13-76.

Boshoff, H. I. \& Mizrahi, v. (1998). Purification, gene cloning, targeted knockout, overexpression, and biochemical characterization of the major pyrazinamidase from Mycobacterium smegmatis. J Bacteriol 180, 5809-5814.

Brennan, P. J. \& Nikaido, H. (1995). The envelope of mycobacteria. Annu Rev Microbiol 64, 29-63.

Butler, R. \& Kilburn, J. O. (1983). Susceptibility of Mycobacterium tuberculosis to pyrazinamide and its relationship to pyrazinamidase activity. Antimicrob Agents Chemother 24, 600-601.

Daffé, M. \& Draper, P. (1998). The envelope layers of mycobacteria with reference to their pathogenicity. Adv Microb Physiol 39, 131-203.

Davies, J. (1994). Inactivation of antibiotics and the dissemination of resistance genes. Science 264, 375-382.

Jackson, M., Raynaud, C., Lanéelle, M.-A., Guilhot, C., LaurentWinter, C., Ensergueix, D., Gicquel, B. \& Daffé, M. (1999). Inactivation of the antigen $85 \mathrm{C}$ gene profoundly affects the mycolate content and alters the permeability of the $M y c o-$ bacterium tuberculosis cell envelope. Mol Microbiol 31, 1573-1587.

Konno, K., Feldmann, F. M. \& McDermott, W. (1967). Pyrazinamide susceptibility and amidase activity of tubercle bacilli. Am Rev Respir Dis 95, 461-469.

Lin, E. C. C. (1976). Glycerol dissimilation and its regulation in bacteria. Annu Rev Microbiol 30, 535-578.

McClatchy, J. K., Tsang, A. Y. \& Cernich, M. S. (1981). Use of pyrazinamidase activity in Mycobacterium tuberculosis as a rapid method for determination of pyrazinamidase susceptibility. Antimicrob Agents Chemother 20, 556-557.

McCune, R. M., Tompsett, R. \& McDermott, W. (1956). The fate of Mycobacterium tuberculosis in mouse tissues as determined by the microbial enumeration technique. II. The conversion of tuberculous infection to the latent state by administration of pyrazinamide and a companion drug. J Exp Med 104, 763-802.

McDermott, W. \& Tompsett, R. (1954). Activation of pyrazinamide and nicotinamide in acidic environments in vitro. Am Rev Tuberc Pulm Dis 70, 748-754.
Mackaness, G. B. (1956). The intracellular activation of pyrazinamide and nicotinamide. Am Rev Tuberc Pulm Dis 74, 718-728.

N'Diaye, E. N., Darzacq, X., Astarie-Dequeker, C., Daffé, M., Calafat, J. \& Maridonneau-Parini, I. (1998). Fusion of azurophil granules with phagosomes and activation of the tyrosine-kinase Hck are specifically inhibited during phagocytosis of mycobacteria by human neutrophils. J Immunol 161, 4983-4991.

Neu, H. C. (1992). The crisis of antibiotic resistance. Science 257, 1064-1073.

Nikaido, H. (1994). Prevention of drug access to bacterial targets : permeability barriers and active efflux. Science 264, 382-388.

Ortalo-Magné, A., Dupont, M.-A., Lemassu, A., Andersen, Å. B., Gounon, P. \& Daffé, M. (1995). Molecular composition of the outermost capsular material of the tubercle bacillus. Microbiology 141, 1609-1620.

Prasad, R., Kalra, V. K. \& Broodie, A. F. (1976). Different mechanisms of energy coupling for transport of various amino acids in cells of Mycobacterium phlei. J Biol Chem 251, 2493-2498.

Quémard, A., Mazère, S., Sut, A., Lanéelle, G. \& Lacave, C. (1995). Certain properties of isoniazid inhibition of mycolic acid synthesis in cell-free systems of M. aurum and M. avium. Biochim Biophys Acta 1254, 98-104.

Raynaud, C., Etienne, G., Peyron, P., Lanéelle, M. A. \& Daffé, M. (1998). Extracellular enzyme activities potentially involved in the pathogenicity of Mycobacterium tuberculosis. Microbiology 144, 577-587.

Sauton, B. (1912). Sur la nutrition minérale du bacille tuberculeux. C R Acad Sci Ser III Sci Vie 155, 860-863.

Scorpio, A. \& Zhang, Y. (1996). Mutations in pncA, a gene encoding pyrazinamidase/nicotinamidase, cause resistance to the antituberculous drug pyrazinamide in tubercle bacillus. Nature Med 6, 662-667.

Scorpio, A., Lindholm-Levy, P., Heifets, L., Gilman, R., Siddiqi, S. Cynamon, M. \& Zhang, Y. (1997). Characterization of pncA mutations in pyrazinamide-resistant Mycobacterium tuberculosis. Antimicrob Agents Chemother 41, 540-543.

Senaratne, R. H., Mobasheri, H., Papavinasasundaram, K. G., Jenner, P., Lea, E. J. A. \& Draper, P. (1998). Expression of a gene for a porin-like protein of the OmpA family from Mycobacterium tuberculosis H37Rv. J Bacteriol 180, 3541-3547.

Spratt, B. G. (1994). Resistance to antibiotics mediated by target alterations. Science 264, 388-393.

Sun, Z., Scorpio, A. \& Zhang, Y. (1997). The pncA gene from naturally pyrazinamide-resistant Mycobacterium avium encodes pyrazinamidase and confers pyrazinamide susceptibility to resistant M. tuberculosis complex organisms. Microbiology 143, 3367-3373.

Tacquet, A., Tison, F., Roos, P. \& Devulder, B. (1967). Activité amidasique des mycobactéries. Technique qualitative nouvelle d'étude en milieu de culture solide. Ann Inst Pasteur (Paris) 112, 378-383.

Tarnok, I. \& Röhrscheidt, E. (1976). Biochemical background of some enzymatic tests used for the differentiation of mycobacteria. Tubercle 57, 145-150.

Tritz, G. J. (1987). NAD biosynthesis and recycling. In Escherichia coli and Salmonella typhimurium: Cellular and Molecular Biology, pp. 557-563. Edited by F. C. Neidhardt and others. Washington, DC: American Society for Microbiology.

Tsukamura, M. \& Tsukamura, J. (1966). Differentiation of mycobacteria by utilization of amides and amino acids as sole nitrogen sources. Am Rev Respir Dis 94, 104-107. 
Wang, L. \& Takayama, K. (1972). Relationship between the uptake of isoniazid and its action on in vivo mycolic acid synthesis in Mycobacterium tuberculosis. Antimicrob Agents Chemother 2, $438-441$.

Wayne, L. G. (1974). Simple pyrazinamidase and urease tests for routine identification of mycobacteria. Am Rev Respir Dis 109, 147-151.

Wayne, L. G. \& Diaz, G. A. (1988). Detection of a novel catalase in extracts of Mycobacterium avium and Mycobacterium intracellulare. Infect Immun 56, 936-941.
Yeager, R. L., Munroe, W. G. \& Dessau, F. I. (1952). Pyrazinamide (aldinamide) in the treatment of pulmonary tuberculosis. Am Rev Tuberc Pulm Dis 65, 523-534.

Youatt, J. \& Tham, S. H. (1969). Radioactive content of $M y c o-$ bacterium tuberculosis after exposure to ${ }^{14} \mathrm{C}$-isoniazid. Am Rev Respir Dis 100, 77-78.

Received 15 September 1998; revised 15 February 1999; accepted 22 February 1999. 